OPEN ACCESS

Edited by:

Michele Marie Kosiewicz, University of Louisville, United States

Reviewed by:

Rebecca Drummond, National Institutes of Health, United States Valerio lebba

Sapienza Università di Roma, Italy

*Correspondence:

Walter J. Lukiw

wlukiw@lsuhsc.edu

Received: 24 March 2017 Accepted: 27 June 2017 Published: 11 July 2017

Citation:

Zhao Y, Jaber V and Lukiw WJ (2017) Secretory Products of the Human GI Tract Microbiome and Their Potential Impact on Alzheimer's Disease (AD): Detection of Lipopolysaccharide (LPS) in AD Hippocampus.

Front. Cell. Infect. Microbiol. 7:318. doi: 10.3389/fcimb.2017.00318

\section{Secretory Products of the Human GI Tract Microbiome and Their Potential Impact on Alzheimer's Disease (AD): Detection of Lipopolysaccharide (LPS) in AD Hippocampus}

\author{
Yuhai Zhao ${ }^{1,2}$, Vivian Jaber ${ }^{1}$ and Walter J. Lukiw ${ }^{1,3,4 *}$ \\ ${ }^{1}$ LSU Neuroscience Center, Louisiana State University Health Science Center, New Orleans, LA, United States, ${ }^{2}$ Department \\ of Anatomy and Cell Biology, Louisiana State University Health Science Center, New Orleans, LA, United States, \\ ${ }^{3}$ Department of Ophthalmology, Louisiana State University Health Science Center, New Orleans, LA, United States, \\ ${ }^{4}$ Department of Neurology, Louisiana State University Health Science Center, New Orleans, LA, United States
}

Although the potential contribution of the human gastrointestinal (Gl) tract microbiome to human health, aging, and disease is becoming increasingly acknowledged, the molecular mechanics and signaling pathways of just how this is accomplished is not wellunderstood. Major bacterial species of the Gl tract, such as the abundant Gram-negative bacilli Bacteroides fragilis (B. fragilis) and Escherichia coli (E. coli), secrete a remarkably complex array of pro-inflammatory neurotoxins which, when released from the confines of the healthy Gl tract, are pathogenic and highly detrimental to the homeostatic function of neurons in the central nervous system (CNS). For the first time here we report the presence of bacterial lipopolysaccharide (LPS) in brain lysates from the hippocampus and superior temporal lobe neocortex of Alzheimer's disease (AD) brains. Mean LPS levels varied from two-fold increases in the neocortex to three-fold increases in the hippocampus, AD over age-matched controls, however some samples from advanced AD hippocampal cases exhibited up to a 26-fold increase in LPS over age-matched controls. This "Perspectives" paper will further highlight some very recent research on Gl tract microbiome signaling to the human CNS, and will update current findings that implicate Gl tract microbiome-derived LPS as an important internal contributor to inflammatory degeneration in the CNS.

Keywords: 42 amino acid amyloid-beta (Aß42) peptide, Alzheimer's disease (AD), Bacteriodetes fragilis (B. fragilis), Escherichia coli (E. coli), lipopolysaccharide (LPS), microbiome, small non-coding RNAs (sncRNAs), thanatomicrobiome

\section{INTRODUCTION: THE HUMAN GI TRACT MICROBIOME}

The human GI tract is fundamentally a highly vascularized and extensively innervated, columnar epithelial-cell lined tube about $9 \mathrm{~m}$ (30 feet) in length that consists of the stomach, small intestine (duodenum, jejunum, and ileum) and large intestine (cecum, colon, rectum, and anal canal; Reinus and Simon, 2014). Each anatomical region of this tubular structure harbors a complex and dynamic microbiome, containing $\sim 1,000$ different species of anaerobic or facultative anaerobic 
bacteria that appear to be characteristic for that GI tract segment. Indeed, the dynamism of the GI tract microbiome along its length is in part reflected by the abundance, speciation, complexity and stoichiometry of individual resident bacterial species. In addition to the major bacterial component of the GI tract are microbial eukaryotes, archaea, fungi, protozoa, viruses, and other commensal microorganisms which make up the remainder. Together with host cells these jointly comprise the complete metaorganism: (i) whose symbiotic associations and interactions are indispensable for homeostatic physiological functions in human health; and (ii) which exhibit alterations in composition in response to dietary factors, developmental stage, GI tract disturbances, aging, and neurological disorders, including $\mathrm{AD}$ (Bhattacharjee and Lukiw, 2013; Hill et al., 2014; Perez et al., 2014; Potgieter et al., 2015; Zhao and Lukiw, 2015; Alkasir et al., 2016; Ghaisas et al., 2016; Hu et al., 2016; Lukiw, 2016; Pistollato et al., 2016; Scheperjans, 2016).

\section{GI TRACT BACTERIAL MICROBIOME-EXUDATES AND SECRETORY PRODUCTS}

Two large prokaryotic classes of Bacteria (or "Eubacteria") and Archaea (or "Archaeobacteria") have been recently reclassified (as of 10/2016) into 35 phyla (http://www.bacterio.net/-classifphyla.html) or major bacterial divisions. Interestingly the GI tract microbiome of Homo sapiens has co-evolved with just two major phyla: Bacteriodetes, which make up $\sim 20 \%$ of all GI tract bacteria, and Firmicutes, which make up $\sim 80 \%$ of all GI tract bacteria; with Actinobacteria ( $\sim 3 \%)$, Proteobacteria $(\sim 1 \%)$, and Verrumicrobia $(\sim 0.1 \%)$ making up significantly smaller fractions. These five bacterial groups appear to constitute the essential "core" of the human GI tract microbiome (http://www.bacterio.net/-classifphyla.html; Zhao et al., 2015; Hug et al., 2016; Lloyd-Price et al., 2016; Sender et al., 2016). The vast proportion of all GI tract microbiota consists of anaerobic or facultative anaerobic bacteria (Bhattacharjee and Lukiw, 2013; Heintz and Mair, 2014; Köhler et al., 2016; Lloyd-Price et al., 2016). For example, although variable, the obligate anaerobe Bacteroides fragilis ( $B$. fragilis; phyla Bacteroidetes) and the facultative anaerobe Escherichia coli (E. coli; phyla Proteobacteria): (i) together constitute $\sim 35-40$ percent of all GI tract bacteria; (ii) are the most abundant Gramnegative bacilli of the middle and lower colon, respectively, of the human GI tract; and (iii) constitute about $\sim 30-50$ percent of the dry weight of fecal matter. B. fragilis or E. coli require about 20 min to divide under optimal conditions of commensal bacterial growth, and unless special biophysical processes of growth dynamics are in operation (such as dormancy, hibernation, spore formation, etc.) have a life span of up to several hours (Choi and Cho, 2016; Pinti et al., 2016; Todar, 2016). Interestingly, species of the obligate anaerobe Bacteroides such as $B$. fragilis display remarkably diverse antibiotic resistance mechanisms and exhibit the highest resistance rates of any anaerobic pathogen. This includes an inherent high-level resistance to penicillin through their ability to produce beta-lactamase enzymes which endow them with multiple resistance to $\beta$-lactam antibiotics such as penicillin and cephamycin (Ayala et al., 2005; Bush and Bradford, 2016; Hu et al., 2016). Specific species of Bacteroidetes such as Bacteroides fragilis ( $B$. fragilis), normally an abundant commensal microorganism of the middle GI tract, are known to be generally beneficial to human health through their ability to digest dietary fiber and related dietary fiber precursors containing substances such as cellulose, lignin, and pectin, which are normally resistant to the action of host digestive enzymes.

Dietary fibers are catabolized into digestible short-chain fatty acids (SCFAs), volatile fatty acids and polysaccharides in part through the biosynthetic capability of this GI tract abundant bacillus (Keenan et al., 2016; Scheperjans, 2016). When $B$. fragilis escapes the highly compartmentalized microbe-dense environment of the GI tract $\left(10^{11}\right.$ microbes per gram of fecal matter), they can induce substantial systemic inflammatory pathology with significant sickness, morbidity and mortality (Choi et al., 2016; Fathi and Wu, 2016; Cattaneo et al., 2017; Shivaji, 2017). Enterotoxigenic strains of $B$. fragilis have been associated with bacteremia, colitis, diarrhea, sepsis, systemic infection, and the development of GI tract cancers and neurological disorders, including $\mathrm{AD}$, that have an increased incidence with aging (Choi et al., 2016; Fathi and $\mathrm{Wu}$, 2016; Keenan et al., 2016; Scheperjans, 2016). Interestingly, certain species of Bacteroidetes have been recently shown to propagate in animal models fed high fat-cholesterol (HFC) diets deprived of sufficient intake of dietary fiber; this suggests that sufficient dietary fiber may have a significant role in regulating the abundance, complexity and stoichiometry of certain species in the GI tract microbiome, including $B$. fragilis (Heinritz et al., 2016; Köhler et al., 2016; Pistollato et al., 2016; unpublished observations). In addition to these positive health benefits however, these vast numbers of human GI tract resident Gram-negative bacilli when stressed secrete prodigious quantities of endotoxins, exotoxins, endotoxins, exotoxins, lipooligosacahrides (LOSs) and lipopolysaccharides (LPSs), amyloids, and small non-coding RNAs (sncRNAs; see below and Figure 1).

\section{ENDOTOXINS AND EXOTOXINS}

Generally, microbiome-derived endotoxins are heat-stable polypeptides associated with the outer membranes of the cell wall of Gram-negative bacteria. They may be composed in part by the Lipid A component of LPS, and once they diffuse into the local environment induce irritation of the GI tract epithelia, capillaries and blood vessels inducing hemorrhage and various pro-inflammatory effects. Endotoxins also induce fever, hemorrhagic shock, diarrhea, altered resistance to bacterial infection, leukopenia followed by leukocytosis, and numerous other systemic effects (Choi et al., 2016; Seong et al., 2016; Zhan and Davies, 2016). For example, in addition to their prodigious LPS generation (see below), B. fragilis endotoxins are a leading cause of anaerobic bacteremia, sepsis and systemic inflammatory distress through their generation of the highly pro-inflammatory zinc metalloproteinase fragilysin, also known as $B$. fragilis toxin 


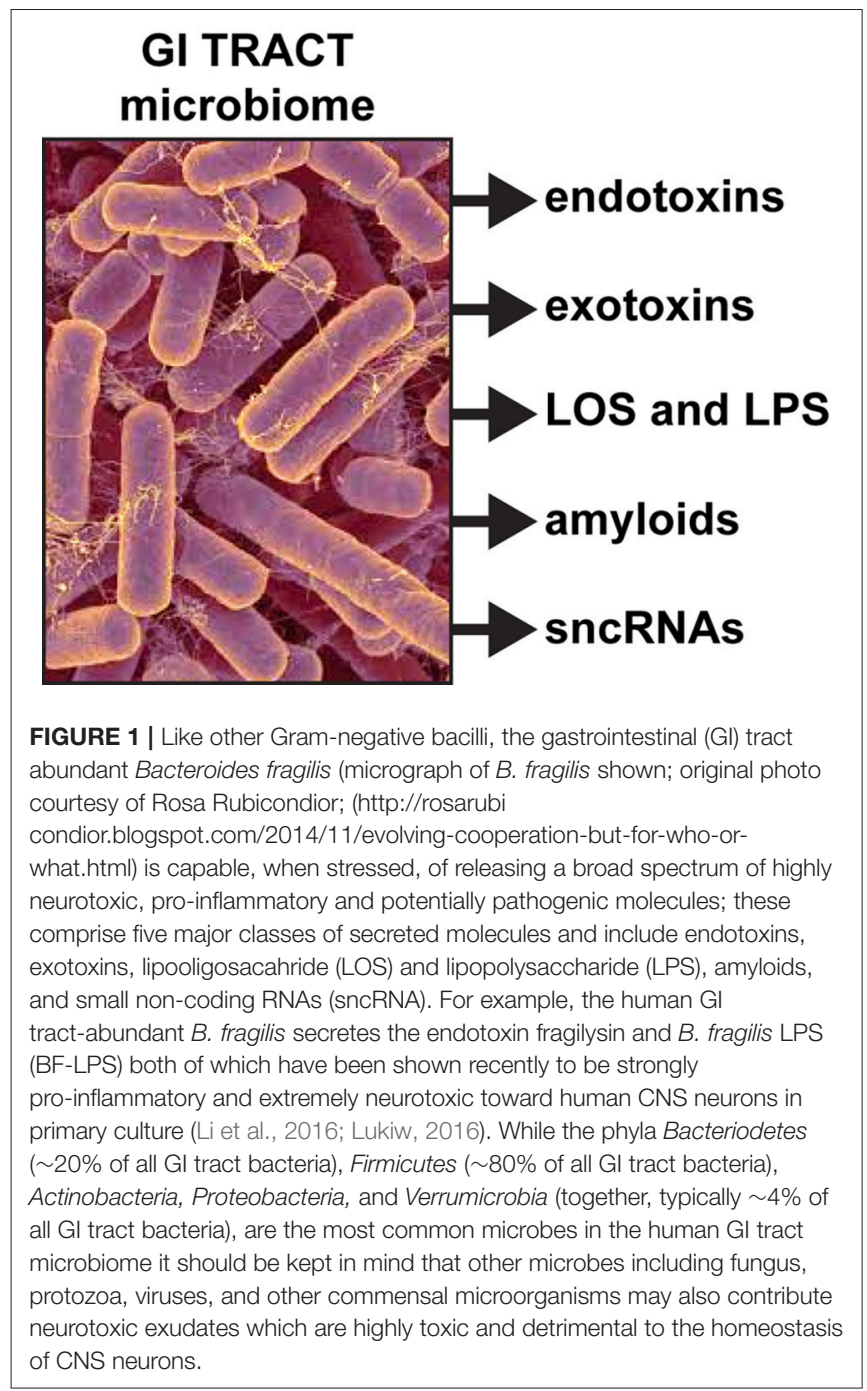

or BFT (Zhao and Lukiw, 2015; Choi et al., 2016; Fathi and $\mathrm{Wu}, 2016)$. BFT has recently been shown to effectively disrupt epithelial cells of GI tract barriers via cleavage of the synaptic type-1 transmembrane zonula adhesion calcium-dependent adhesion protein E-cadherin (Choi et al., 2016; Seong et al., 2016; Zhan and Davies, 2016). It is currently not understood if GI tract- or BBB-disrupting proteolytic endotoxins such as BFT are able to propagate their pathogenic activities via the systemic circulation to further disrupt the GI tract or BBB at distant sites, to ultimately transfer endotoxins, exotoxins, LPSs, amyloids and/or sncRNAs into the cerebrovascular circulation to target brain cells within the CNS. B. fragilis has been suggested to contribute to neurodevelopmental pathology in autism spectrum disorder (ASD; Hsiao et al., 2013; Hofer, 2014; Keaney and Campbell, 2015). It has also recently been reported that along with BFTs amyloid peptide-dependent changes in synaptic adhesion affect both the function and integrity of synapses, suggesting that the observed failure of synaptic adhesion in $\mathrm{AD}$ play key roles in the progressive disruption of functional signaling throughout neuronal networks, as is observed in $\mathrm{AD}$ brain (Lin et al., 2014; Seong et al., 2015; Leshchyns'ka and Sytnyk, 2016).

Exotoxins are generally complex soluble polypeptides produced on the inside of pathogenic bacteria as part of their normal growth and metabolism, and these are typically excreted by living cells or released during bacterial cell lysis into the surrounding medium. The relatively short lifespan of GI tract bacteria (see above) and their subsequent lysis indicate that lysed bacteria contents may be a relatively persistent source of exotoxins which may need to be either efficiently neutralized or eliminated by the GI tract. Interestingly, under some conditions in rodents certain endotoxins are so toxic that they may be lethal to the host before the innate immune system has a chance to mount immune defenses to promote their neutralization (Bhattacharjee and Lukiw, 2013; Asti and Gioglio, 2014; Hill et al., 2014; Hill and Lukiw, 2015).

\section{LIPOOLIGOSACAHRIDE (LOS) AND LIPOPOLYSACCHARIDE (LPS)}

As an abundant Gram negative bacilli of the human GI tract microbiome both $B$. fragilis and $E$. coli secrete lipooligosacahrides (LOS) and lipopolysaccharides (LPS) that are strongly immunogenic and highly pro-inflammatory toward human neurons (Bian et al., 2011; Alkasir et al., 2016; Fathi and Wu, 2016; Foster et al., 2016; Ghaisas et al., 2016; Hug et al., 2016; Lukiw, 2016; Rogers and Aronoff, 2016; Sender et al., 2016; Sharon et al., 2016). LPSs, as characteristic components of the outer leaflet of the outer membrane of Gram-negative bacteria shed into the extracellular space, play key roles in host-pathogen interactions and the innate-immune system (Hill and Lukiw, 2015; Zhao et al., 2015; Maldonado et al., 2016). While LPSs contain large and hypervariable oligosaccharide/polysaccharide regions, the relatively conserved lipid region (lipid A) is the endotoxic and biologically active moiety that is largely responsible for septic shock (Jiang et al., 2016; Maldonado et al., 2016). A canonic LPS structure is represented by that of E. coli LPS, one of the most potent neurotoxic lipid A species known, consisting of a 1,4'-biphosphorylated glucosamine disaccharide bearing six fatty acids which are unbranched chains 12-14 methyl(ene) units in length. Other "lipid A" species show variability in the number, length, and composition of the attached fatty acids, as well as variability in the degree of phosphorylation and number and types of substituted phosphate ligands. For instance, BF-LPS lipid A is penta-acylated and mono-phosphorylated, and contains branched fatty acids 15-17 methyl(ene) units in length; deviations from the canonical lipid A structure are known to have a profound impact on innate-immune responses. Gram-negative bacterial exudates such as BF-LPSs are hypervariable in composition, and different Bacteroidetes species appear to generate unique temporal patterns of LPS production. These exhibit rapid and remarkably adaptive changes in LPS structure and alterations in damageor pathogen-associated molecular patterns (DAMP/PAMP) as strategies for host immune evasion (Friedland, 2015; Land, 2015; Maldonado et al., 2016; Richards et al., 2016). Here, for 
the first time, we provide evidence that E. coli LPS is abundant in neocortical and hippocampal extracts from $\mathrm{AD}$ brain, regions of the human limbic system targeted by intense neuroinflammation characteristic of the $\mathrm{AD}$ process (see Figure 1 and legend). Similarly the pathological actions of LPS on the induction of pro-inflammatory signaling in primary human neurons have recently been demonstrated, and additional studies are in progress (Lukiw, 2016).

\section{AMYLOIDS}

Atypical amyloid generation, aggregation, folding, and impaired clearance are characteristic pathological features of human neuro-inflammatory and neurodegenerative disorders of the CNS that include AD (Calsolaro and Edison, 2016; Andreeva et al., 2017). What is generally not appreciated is that a major secretory product of the GI tract microbiome is amyloid, and that the life-long contribution of microbial amyloid to CNS pathophysiology can be very substantial. "Amyloid" is a generic term for any aggregated, insoluble, lipoprotein-enriched deposit that exhibits $\beta$-pleated sheet structures oriented perpendicular to the fibrillar axis (Lukiw, 2012; Clark and Vissel, 2015; Lim et al., 2015; Andreeva et al., 2017; Bolós et al., 2017). The potential for amyloid formation is surprisingly high in almost all proteins; a major factor for amyloid formation is the presence within proteins of primary amino acid sequences that can form a tight, self-complementary interface with an identical segment, thus permitting the cooperative formation of a steric zipper. Two self-complementary beta-sheets form the backbone of the amyloid fibril (Goldschmidt et al., 2010; Buxbaum and Linke, 2012; Andreeva et al., 2017). The characterization of the "amylome," a categorization of amino acid sequences that possess self-complementary interfaces and high fiber-forming propensity has improved our understanding of the capability of different proteins to generate amyloid (Goldschmidt et al., 2010; Lukiw, 2012; Andreeva et al., 2017). The progressive generation and aggregation of amyloids contribute to "dense-deposit" disease; the pathogenesis of diseases that accumulate amyloid, including $\mathrm{AD}$, all involve prominent inflammatory responses at sites of amyloid deposition-these accumulations are often mediated by microglial cells, the "resident immune cells" of the CNS. Interestingly, most microbial species, including fungi and bacteria, secrete self-associating and strongly amyloidogenic lipoproteins (Hill et al., 2014; Syed and Boles, 2014; Schwartz et al., 2016). For instance, amyloids are associated with fungal surface-structures and the recent observation of amyloidogenic fungal proteins and diffuse mycoses in the blood of $\mathrm{AD}$ patients suggest that chronic fungal infection over the course of aging may increase AD risk (Alonso et al., 2014; Hill et al., 2014). Of further relevance is that: (i) $\mathrm{A} \beta 42$ peptide monomers, dimers, oligomers and fibrils each induce patterns of pro-inflammatory gene signaling typical of the classical microglial-mediated innateimmune and inflammatory response induced by infectious agents such as bacterial LPS (Ferrera et al., 2014; Calsolaro and Edison, 2016; Lukiw, 2016; Andreeva et al., 2017); (ii) the presence of bacterial LPS or endotoxin/exotoxin-mediated inflammatory signaling strongly contributes to amyloid neurotoxicity (Lee et al., 2008; Asti and Gioglio, 2014; Zhao and Lukiw, 2015;
Zhao et al., 2016); (iii) AD amyloids, like prion amyloids, once formed, may induce a self-perpetuating process leading to amplification, aggregation, and spreading of pathological aggregates (Le et al., 2014); and (iv) recently it has been shown that $A \beta 42$ peptide fibrillogenesis is strongly potentiated by soluble bacterial exudates and viruses such as HSV-1, suggesting the contribution of microbial-sourced factors and/or infectious events to amyloidogenesis, a distinguishing feature of the $\mathrm{AD}$ neuropathology (Hill et al., 2014; Stilling et al., 2014; Zhao et al., 2015; Russo et al., 2017).

\section{SMALL NON-CODING RNA (sncRNA)}

While the secretion of proteins, lipids, and nucleic acids (both RNA and DNA) from neural cells into the extracellular space is a commonly recognized phenomenon in neurobiology, the secretion of small non-coding RNA (sncRNA) from microbial cells into the GI tract has only been very recently characterized (Ghosal et al., 2015; Lukiw, 2016; Ghosal, 2017). Employing multi-component secretion systems, sncRNAs may be exuded from bacteria as separate entities, or more commonly, contained within lipid spheres or outer membrane vesicles (OMVs; Ghosal et al., 2015; unpublished observations). A major fraction of all secreted extracellular RNAs are sncRNAs in the size range of 15-40 nucleotides derived from specific intracellular bacterial RNAs. These sncRNAs have been speculated to be involved in immune-evasion, intra-species communication, in inter-kingdom genetic exchanges, pathogenicity and/or microbiome-host signaling; indeed protein-, lipid-, and nucleic acid-containing OMVs released by GI tract Gram-negative bacteria can be intensely pro-inflammatory, pathogenic or even lethal to the host (Zhao and Lukiw, 2015; Ghosal, 2017; Lukiw and Rogaev, 2017; unpublished observations). Several important questions remain to be answered: (i) do secreted sncRNAs play any role in microbiome survival, immune evasion and/or antibiotic resistance? (ii) how do GI tract microbes promote and organize the regulation of sncRNA trafficking (iii) how are bacterial sncRNAs transported across bacterial membranes and subsequently released into the extracellular space? (iv) how are the sncRNAs selected and packaged for export? and (v) are there differences in secreted sncRNA profiles between pathogenic and non-pathogenic bacteria and/or between healthy and diseased states of the host? Further investigations in the field of extracellular bacterial sncRNAs are clearly needed to shed light on their potential role as mediators of microbiomehost signaling and intercellular communication. By studying bacterial secreted sncRNA patterns, we may be able to further advance our understanding of the complex interactions that exist between humans and their GI tract microbiome and design, perhaps through dietary manipulation, highly effective intervention strategies that could improve and optimize human neurological health.

\section{THANATOMICROBIOME}

Evidence for the immense biophysiological efforts in keeping the GI tract microbiome contained within GI tract compartments 
and from expansion beyond its normal niche, comes from analysis of the human microbiome at the time of death. Very little data are available concerning what happens to the microbiome when a human host dies-in a healthy adult, most internal organs such as the spleen, liver, heart, and brain are generally devoid of microbes because the innate-immune system or other microbial components keeps them in check. After death, however, the generation of ATP ceases, the innate-immune system falters and microbes proliferate throughout the body; this has recently been shown to begin in the ileocecal area of the GI tract, spreading to the liver and spleen, and continuing to the heart and brain (Alan and Sarah, 2012; Can et al., 2014; Clement et al., 2016; Javan et al., 2016). Still evolving concepts of what happens to GI tract microbiome speciation and complexity at the time of death are currently being researched. Indeed the thanatomicrobiome (thanatos, Greek for death) is a relatively new designation defined as the composition and organization of the GI tract microbiome and other microbial communities following cessation of all life activities (Clement et al., 2016; Javan et al., 2016). Recent studies so far underscore the fact that in the GI tract microbiome there is a constant struggle to contain GI tract microbiome integrity and regulate specific bacterial abundance and complexity (Clement et al., 2016; Javan et al., 2016). Ongoing work from temporal studies on the thanatomicrobiome across defined post-mortem intervals (PMI) further indicate (i) that the majority of the microbes within the human body and those which propagate most rapidly at the time of death are the obligate anaerobes that begin to non-randomly proliferate from the GI tract continuing throughout the human organs over the PMI (Javan et al., 2016); and (ii) that comprehensive knowledge of the number and abundance of each organ's microbial signature could be useful to forensic microbiologists as a new source of data for estimating PMI. These data combined with nucleic acid sequencing and bioinformatics would also be invaluable in aiding researchers who use post-mortem tissues in their research work and in forensic criminology, microbial speciation and the study of microbiome-host genetics in the later stages of life.

\section{CONCLUDING REMARKS}

In summary, the human GI tract constitutes the largest repository of the human microbiome, and its impact on human neurological aging, health and disease is becoming increasingly appreciated. Consisting of about $\sim 4 \times 10^{13}$ microorganisms, the human GI tract microbiome forms a highly complex, symbiotic and dynamic ecosystem within the host and dietary factors and host genetics appear to have a strong influence on microbial abundance, speciation and complexity, and their ability to influence CNS functions (Foster et al., 2016; Li et al., 2016; Richards et al., 2016; Brandscheid et al., 2017; Tremlett et al., 2017). We sincerely hope that this "Perspectives" article has effectively highlighted recent findings on microbial-derived endotoxins, exotoxins, LOSs and LPSs, amyloids and sncRNAs and has stimulated interest in the potential contribution of these neurotoxic and pro-inflammatory microbial exudates to agerelated inflammatory neurodegeneration, amyloidogenesis, and
AD-relevant pathology (Figure 1). Taken together, these current observations and recent data advance at least seven areas in our understanding of the role of the GI tract microbiome in age-related neurological diseases associated with progressive, inflammatory neurodegeneration of the human brain: (i) that the GI tract microbiome are a potent source of neurotoxic species that are abundantly secreted by multiple Gram-negative bacilli in the gut (B. fragilis, E. coli, and others); (ii) that bacterial LPS are readily detectable in the neocortex and hippocampus of the $\mathrm{AD}$ brain, and at significantly higher abundance in $\mathrm{AD}$ than controls, indicating that LPS may be able to transit physiological barriers to access CNS compartments (Figure 2); (iii) that the transit of highly pro-inflammatory neurotoxins such as LPS across compromised GI tract and blood-brain barriers underscore the critical roles of cellular adhesion structures in allowing passage of noxious molecules from the GI tract into the systemic circulation and CNS (Montagne et al., 2016; Soenen et al., 2016; van de Haar et al., 2016); (iv) that extremely complex mixtures of neurotoxins may be generated by either single microbes or by combinations of bacilli that constitute the GI tract microbiome (Figure 1); (v) that biophysical, gastrointestinal, and neurobiological barriers that may become more "leaky" with aging again underscore the important role of intact membrane barriers in moderating systemic and CNS inflammation and immune-mediated inflammatory disease (Hill and Lukiw, 2015; Keaney and Campbell, 2015; Montagne et al., 2015; Choi et al., 2016; Köhler et al., 2016; Minter et al., 2016a; Richards et al., 2016; van de Haar et al., 2016; Zhan and Davies, 2016; Varatharaj and Galea, 2017); (vi) that bacterial complexity, neurotoxin abundance, speciation, and complexity in the CSF, blood serum or in brain tissues may be useful for the diagnosis of $\mathrm{AD}$ (Zhao et al., 2015; Soenen et al., 2016); and (vii) that studies on the thanatomicrobiome should be useful for a clearer understanding of the neuro- and micro-biological processes in operation over the PMI that should be useful in scientific research that utilizes post-mortem tissues in basic research, in forensic applications, in criminology and in the more accurate diagnosis of neurological disease (Clement et al., 2016; Javan et al., 2016). While one other recent investigation reported the detection of LPS in gray matter (temporal lobe) and white matter (frontal lobe) in $\mathrm{AD}$ (Zhan et al., 2016), here for the first time we report the detection of bacterial LPS in brain lysates from AD hippocampus, an anatomical region of the $\mathrm{AD}$ brain that develops the earliest and most profound neuropathology. Some advanced AD hippocampal patients exhibited up to a 26 -fold increase in LPS over age-matched controls. Lastly, more research into the intriguing field of human GI tract microbiome-host interaction and its potential contributory role to human aging, neurological health and disease is clearly needed. The study of these symbiotic prokaryotic and eukaryotic divisions, their evolution and their intriguing interrelationships, genetic interactions and associations in future work should be useful in expanding our understanding of microbiome-host interplay and control in the initiation, development, propagation, and diagnosis of human neurological disorders in which microbial involvement appears to play some contributory or even deterministic role. 


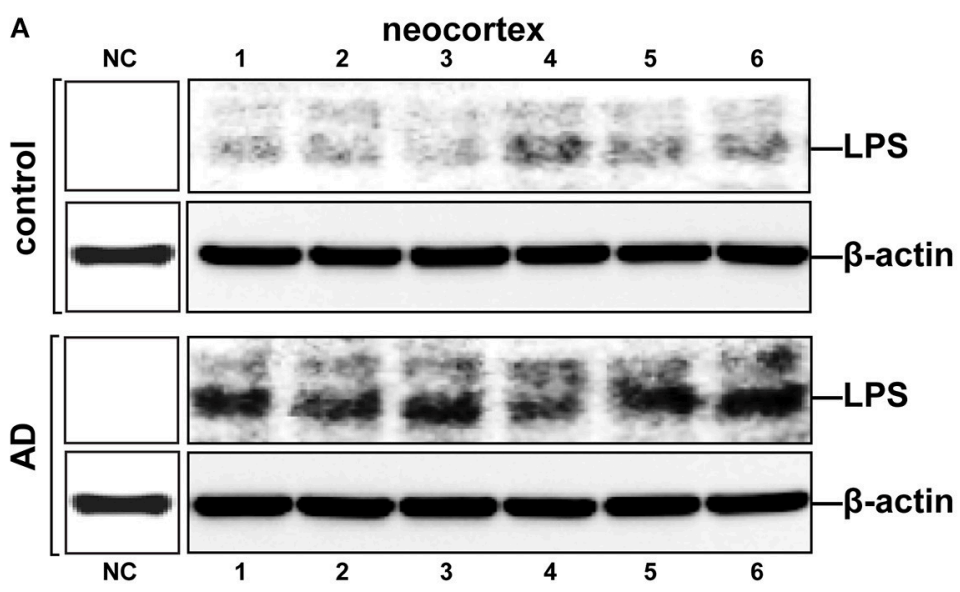

C
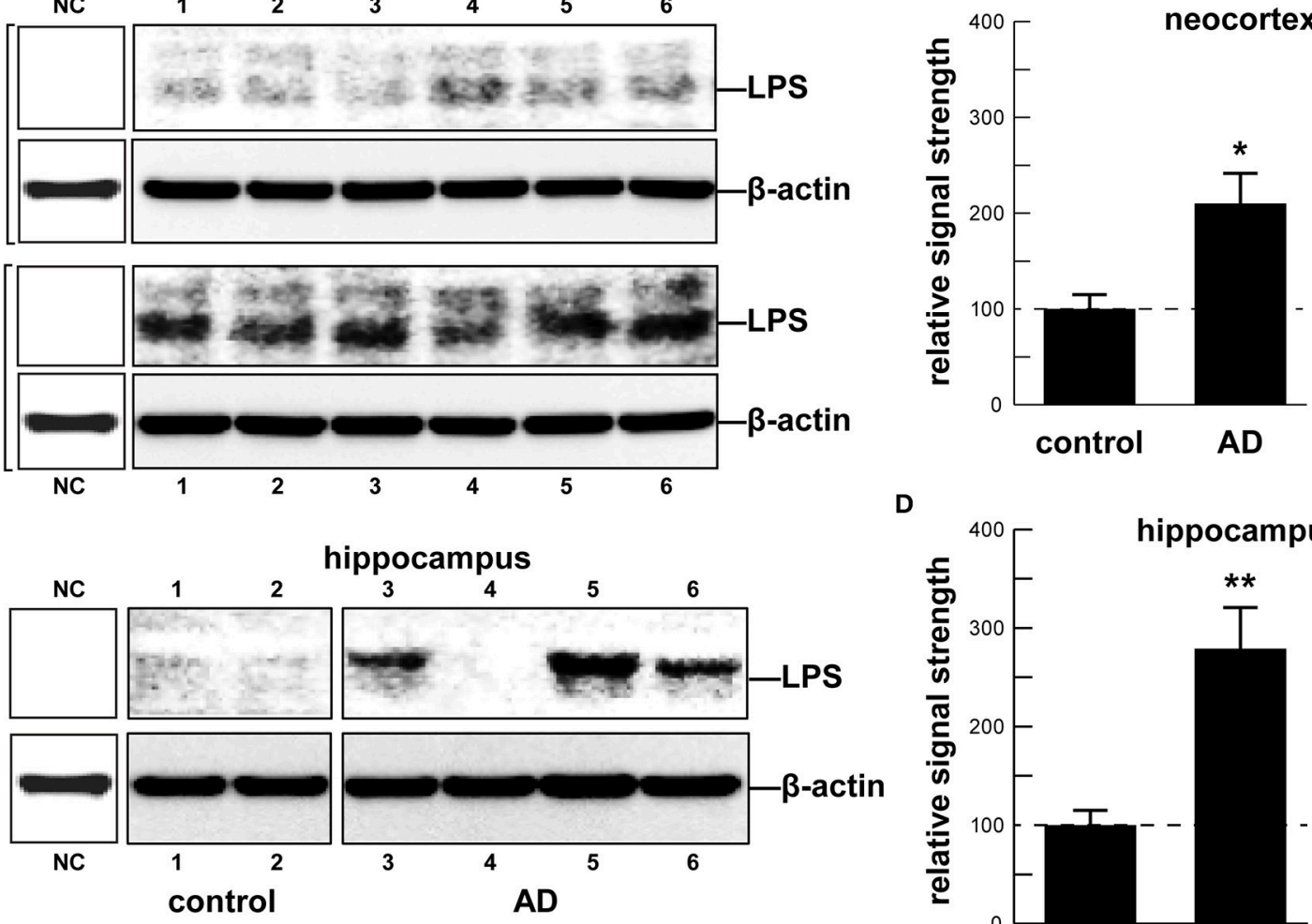

D

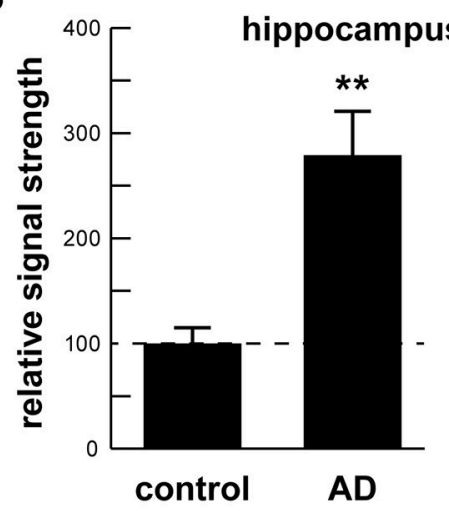

FIGURE 2 | (A) human brain temporal lobe neocortex [ $N=6$ control and 6 sporadic AD cases; quantified in (B)]; and (C) hippocampus ( $N=2$ control and $N=4$ AD cases; quantified in (D)] were analyzed for LPS against $\beta$-actin abundance in the same sample (using anti-E. coli LPS; cat \# ab35654 from Abcam, Cambridge UK and anti- $\beta$-actin cat \# 3700 from Cell Signaling, Danvers MA, USA) using Western analysis as previously described by our group (Bhattacharjee et al., 2016; Zhao et al., 2016); all AD and control tissues were analyzed in a RNA-analysis clean room facility; all control and AD tissues were age- and gender-matched; there were no significant differences between the age (control $72.9 \pm 8.1$ years, AD 74.2 \pm 9.1 years), gender (all female), PMl (all tissues $3.5 \mathrm{~h}$ post-mortem or less), RNA quality or RNA yield between each of the two groups; LPS abundance was found to be on average over two-fold as abundant in AD when compared to age-, gender, and PMI-matched control neocortex in 6 of 6 cases; LPS was found to be on average three-fold as abundant in AD when compared to age-, gender, and PMI-matched control hippocampus in 3 of 4 cases; some advanced AD hippocampal samples exhibited up to a 26-fold increase in LPS over age-matched controls (C, LPS in control lane 2 vs. AD lane 5); because one major source of LPS are Gram-negative bacteria of the human Gl tract (predominantly $B$. fragilis and $E$. coli), this suggests that in vivo intensely pro-inflammatory LPS species may be able to "leak" through at least two major biophysiological barriers -the Gl tract barrier and the BBB - to access brain compartments (see Devier et al., 2015; Halmer et al., 2015; Choi et al., 2016; Minter et al., 2016b; Montagne et al., 2016; Richards et al., 2016; Soenen et al., 2016; van de Haar et al., 2016; Zhan and Davies, 2016; Zhao et al., 2016; Varatharaj and Galea, 2017). Unpublished work from this laboratory further indicates the positive detection of LPS in 36 of 36 AD tissues sampled from the superior temporal lobe neocortex in aged individuals (age range 66-79 yr; see Table 1 in Cui et al., 2010). Another recent investigation reports the finding of LPS in gray matter (temporal lobe) and white matter (frontal lobe) of the AD brain (Zhan et al., 2016). Together these data also suggest that neurotoxic cocktails secreted by multiple Gl tract microbes or other microbial species (Figure 1) may have considerable potential to support intense pro-inflammatory signaling within the CNS especially over the course of aging when barriers become more "leaky" (Hill and Lukiw, 2015; Keaney and Campbell, 2015; Montagne et al., 2015; Choi et al., 2016; Köhler et al., 2016; Minter et al., 2016a,b; Richards et al., 2016; van de Haar et al., 2016; Zhan and Davies, 2016; Varatharaj and Galea, 2017); (B) and (D) represent the mean plus one standard deviation of that mean; ${ }^{*} p<0.05,{ }^{* *} p<0.01$ ANOVA; NC, negative control using a control murine brain extract (strain C57BL/6J); in (B) and (D) a dashed horizontal line at 100 is included for ease of comparison.

\section{AUTHOR CONTRIBUTIONS}

YZ and VJ analyzed brains for LPS content; WL compiled and analyzed the data and wrote the paper.

\section{ACKNOWLEDGMENTS}

This research work was presented in part at the Society for Neuroscience (SFN) Annual Meeting 12-16 November 2016, San Diego CA, USA and at the Alzheimer Association International
Congress 2016 (AAIC 2016) Annual conference 21-27 July 2016 in Toronto, Canada. These studies utilized total nucleic acid and/or cytoplasmic fractions extracted from primary human neuronal-glial (HNG) co-cultures; sincere thanks are extended to Drs. P. N. Alexandrov, J. G. Cui, F. Culicchia, W. Poon, K. Navel, C. Hebel, and C. Eicken for short PMI human brain tissues or extracts, unpublished Western data and immunochemistry, HNG tissue culture and NF-kB-DNA binding assay, initial bioinformatics and data interpretation, and to D. Guillot and A. I. Pogue for expert technical assistance and medical 
artwork. All human tissues were used in strict accordance with ethical compliance procedures and protocols followed by donor institutions; thanks are also extended to the Institute for Memory Impairments and Neurological Disorders (MIND), to the University of California at Irvine (UCI) and to the many neuropathologists, physicians and researchers of the US, Canada and Europe who have provided high quality, short PMI human CNS or extracted tissue fractions for scientific study. Research

\section{REFERENCES}

Alan, G., and Sarah, J. P. (2012). Microbes as forensic indicators. Trop. Biomed. 29, 311-330.

Alkasir, R., Li, J., Li, X., Jin, M., and Zhu, B. (2016). Human gut microbiota: the links with dementia development. Protein Cell 8, 90-102. doi: 10.1007/s13238-016-0338-6

Alonso, R., Pisa, D., Marina, A. I., Morato, E., Rábano, A., and Carrasco, L. (2014). Fungal infection in patients with Alzheimer's disease. J. Alzheimers Dis. 41, 301-311. doi: 10.3233/JAD-132681

Andreeva, T. V., Lukiw, W. J., and Rogaev, E. I. (2017). Biological basis for amyloidogenesis in Alzheimer's disease. Biochemistry 82, 122-139. doi: 10.1134/S0006297917020043

Asti, A., and Gioglio, L. (2014). Can a bacterial endotoxin be a key factor in the kinetics of amyloid fibril formation? J. Alzheimers Dis. 39, 169-179. doi: 10.3233/JAD-131394

Ayala, J., Quesada, A., Vadillo, S., Criado, J., and Píriz, S. (2005). Penicillin-binding proteins of Bacteroides fragilis and their role in the resistance to imipenem of clinical isolates. J. Med. Microbiol. 54, 1055-1064. doi: 10.1099/jmm.0.45930-0

Bhattacharjee, S., and Lukiw, W. J. (2013). Alzheimer's disease and the microbiome. Front. Cell. Neurosci. 7:153. doi: 10.3389/fncel.2013.00153

Bhattacharjee, S., Zhao, Y., Dua, P., Rogaev, E. I., and Lukiw, W. J. (2016). miRNA34a-mediated down-regulation of the microglial-enriched triggering receptor and phagocytosis-sensor TREM2 in age-related macular degeneration. PLoS ONE 11:e0150211. doi: 10.1371/journal.pone.0150211

Bian, Z. M., Elner, S. G., Khanna, H., Murga-Zamalloa, C. A., Patil, S., and Elner, V. M. (2011). Expression and functional roles of caspase-5 in inflammatory responses of human retinal pigment epithelial cells. Invest. Ophthalmol. Vis. Sci. 52, 8646-8656. doi: 10.1167/iovs.11-7570

Bolós, M., Perea, J. R., and Avila, J. (2017). Alzheimer's disease as an inflammatory disease. Biomol. Concepts 8, 37-43. doi: 10.1515/bmc-2016-0029

Brandscheid, C., Schuck, F., Reinhardt, S., Schäfer, K. H., Pietrzik, C. U., and Grimm, M., et al. (2017). Altered gut microbiome composition and tryptic activity of the 5xFAD Alzheimer's mouse model. J. Alzheimers Dis. 56, 775-788. doi: 10.3233/JAD-160926

Bush, K., and Bradford, P. A. (2016). $\beta$-Lactams and $\beta$-Lactamase inhibitors: an overview. Cold Spring Harb. Perspect. Med. 6:a025247. doi: $10.1101 /$ cshperspect.a025247

Buxbaum, J. N., and Linke, R. P. (2012). A molecular history of the amyloidoses. J. Mol. Biol. 421, 142-159. doi: 10.1016/j.jmb.2012.01.024

Calsolaro, V., and Edison, P. (2016). Neuroinflammation in Alzheimer's disease: Current evidence and future directions. Alzheimers Dement. 12, 719-732. doi: 10.1016/j.jalz.2016.02.010

Can, I., Javan, G. T., Pozhitkov, A. E., and Noble, P. A. (2014). Distinctive thanatomicrobiome signatures found in the blood and internal organs of humans. J. Microbiol. Methods 106, 1-7. doi: 10.1016/j.mimet.2014.07.026

Cattaneo, A., Cattane, N., Galluzzi, S., Provasi, S., Lopizzo, N., Festari, C., et al. (2017). Association of brain amyloidosis with pro-inflammatory gut bacterial taxa and peripheral inflammation markers in cognitively impaired elderly. Neurobiol. Aging. 49, 60-68. doi: 10.1016/j.neurobiolaging.2016.08.019

Choi, H. H., and Cho, Y. S. (2016). Fecal microbiota transplantation: current applications, effectiveness, and future perspectives. Clin. Endosc. 49, 257-265. doi: $10.5946 /$ ce.2015.117

Choi, V. M., Herrou, J., Hecht, A. L., Teoh, W. P., Turner, J. R., Crosson, S., et al. (2016). Activation of Bacteroides fragilis toxin by a novel bacterial on the human microbiome, pro-inflammatory and pathogenic signaling in the Lukiw laboratory involving the innate-immune response, neuroinflammation and amyloidogenesis in $\mathrm{AD}$ and in other neurological diseases was supported through an unrestricted grant to the LSU Eye Center from Research to Prevent Blindness (RPB); the Louisiana Biotechnology Research Network (LBRN) and NIH grants NEI EY006311, NIA AG18031 and NIA AG038834.

protease contributes to anaerobic sepsis in mice. Nat. Med. 22, 563-567. doi: $10.1038 / \mathrm{nm} .4077$

Clark, I. A., and Vissel, B. (2015). Amyloid $\beta$ : one of three dangerassociated molecules that are secondary inducers of the proinflammatory cytokines that mediate Alzheimer's disease. Br. J. Pharmacol. 172, 3714-3727. doi: $10.1111 / \mathrm{bph} .13181$

Clement, C., Hill, J. M., Dua, P., Culicchia, F., and Lukiw, W. J. (2016). Analysis of RNA from Alzheimer's disease post-mortem brain tissues. Mol. Neurobiol. 53, 1322-1328. doi: 10.1007/s12035-015-9105-6

Cui, J. G., Li, Y. Y., Zhao, Y., Bhattacharjee, S., and Lukiw, W. J. (2010). Differential regulation of interleukin-1 receptor-associated kinase-1 (IRAK-1) and IRAK-2 by microRNA-146a and NF-kB in stressed human astroglial cells and in Alzheimer disease. J. Biol. Chem. 285, 38951-38960. doi: 10.1074/jbc.M110.178848

Devier, D. J., Lovera, J. F., and Lukiw, W. J. (2015). Increase in NF-кB-sensitive miRNA-146a and miRNA-155 in multiple sclerosis (MS) and pro-inflammatory neurodegeneration. Front. Mol. Neurosci. 8:5. doi: 10.3389/fnmol.20 15.00005

Fathi, P., and $\mathrm{Wu}, \mathrm{S}$. (2016). Isolation, detection and characterization of enterotoxigenic Bacteroides fragilis in clinical samples. Open Microbiol. J. 10, 57-63. doi: 10.2174/1874285801610010057

Ferrera, D., Mazzaro, N., Canale, C., and Gasparini, L. (2014). Resting microglia react to $\mathrm{A} \beta 42$ fibrils but do not detect oligomers or oligomer-induced neuronal damage. Neurobiol. Aging. 35, 2444-2457. doi: 10.1016/j.neurobiolaging.2014.05.023

Foster, J. A., Lyte, M., Meyer, E., and Cryan, J. F. (2016). Gut microbiota and brain function: an evolving field in neuroscience. Int. J. Neuropsychopharmacol. 19:pyv114. doi: 10.1093/ijnp/pyv114

Friedland, R. P. (2015). Mechanisms of molecular mimicry involving the microbiota in neurodegeneration. J. Alzheimers Dis. 45, 349-362. doi: 10.3233/JAD-142841

Ghaisas, S., Maher, J., and Kanthasamy, A. (2016). Gut microbiome in health and disease: Linking the microbiome-gut-brain axis and environmental factors in the pathogenesis of systemic and neurodegenerative diseases. Pharmacol Ther. 158, 52-62. doi: 10.1016/j.pharmthera.2015.11.012

Ghosal, A. (2017). Importance of secreted bacterial RNA in bacterialhost interactions in the gut. Microb. Pathog. 104, 161-163. doi: 10.1016/j.micpath.2017.01.032

Ghosal, A., Upadhyaya, B. B., Fritz, J. V., Heintz-Buschart, A., Desai, M. S., Yusuf, D., et al. (2015). The extracellular RNA complement of Escherichia coli. Microbiologyopen 4, 252-266. doi: 10.1002/mbo3.235

Goldschmidt, L., Teng, P. K., Riek, R., and Eisenberg, D. (2010). Identifying the amylome, proteins capable of forming amyloid-like fibrils. Proc. Natl. Acad. Sci. U.S.A. 107, 3487-3492. doi: 10.1073/pnas.0915166107

Halmer, R., Davies, L., Liu, Y., Fassbender, K., and Walter, S. (2015). The innate immune receptor CD14 mediates lymphocyte migration in EAE. Cell. Physiol. Biochem. 37, 269-275. doi: 10.1159/000430351

Heinritz, S. N., Weiss, E., Eklund, M., Aumiller, T., Heyer, C. M., Messner, S., et al. (2016). Impact of a high-fat or high-fiber diet on intestinal microbiota and metabolic markers in a pig model. Nutrients 8:E317. doi: 10.3390/nu8050317

Heintz, C., and Mair, W. (2014). You are what you host: microbiome modulation of the aging process. Cell 156, 408-411. doi: 10.1016/j.cell.2014.01.025

Hill, J. M., and Lukiw, W. J. (2015). Microbial-generated amyloids and Alzheimer's disease (AD). Front. Aging Neurosci. 7:9. doi: 10.3389/fnagi.2015. 00009 
Hill, J. M., Clement, C., Pogue, A. I., Bhattacharjee, S., Zhao, Y., and Lukiw, W J. (2014). Pathogenic microbes, the microbiome, and Alzheimer's disease (AD). Front. Aging Neurosci. 6:127. doi: 10.3389/fnagi.2014.00127

Hofer, U. (2014). Microbiome: B. fragilis and the brain. Nat. Rev. Microbiol. 12, 76-77. doi: 10.1038/nrmicro3197

Hsiao, E. Y., McBride, S. W., Hsien, S., Sharon, G., Hyde, E. R., McCue, T., et al. (2013). Microbiota modulate behavioral and physiological abnormalities associated with neurodevelopmental disorders. Cell 155, 1451-1463. doi: 10.1016/j.cell.2013.11.024

Hu, X., Wang, T., and Jin, F. (2016). Alzheimer's disease and gut microbiota. Sci. China Life Sci. 59, 1006-1023. doi: 10.1007/s11427-016-5083-9

Hug, L. A., Baker, B. J., Anantharaman, K., Brown, C. T., Probst, A. J., Castelle, C. J., et al. (2016). A new view of the tree of life. Nat. Microbiol. 1:16048. doi: $10.1038 /$ nmicrobiol.2016.48

Javan, G. T., Finley, S. J., Abidin, Z., and Mulle, J. G. (2016). The thanatomicrobiome: a missing piece of the microbial puzzle of death. Front. Microbiol. 7:225. doi: 10.3389/fmicb.2016.00225

Jiang, Q., Jin, S., Jiang, Y., Liao, M., Feng, R., Zhang, L., et al. (2016). Alzheimer's disease variants with the genome-wide significance are significantly enriched in immune pathways and active in immune cells. Mol. Neurobiol. 54, 594-600. doi: $10.1007 /$ s12035-015-9670-8

Keaney, J., and Campbell, M. (2015). The dynamic blood-brain barrier. FEBS J. 282, 4067-4079. doi: 10.1111/febs.13412

Keenan, J. I., Aitchison, A., Purcell, R. V., Greenlees, R., Pearson, J. F., and Frizelle, F. A. (2016). Screening for enterotoxigenic Bacteroides fragilis in stool samples. Anaerobe 40, 50-53. doi: 10.1016/j.anaerobe.2016.05.004

Köhler, C. A., Maes, M., Slyepchenko, A., Berk, M., Solmi, M., Lanctôt, K. L., et al. (2016). The gut-brain axis, including the microbiome, leaky gut and bacterial translocation: mechanisms and pathophysiological role in Alzheimer's disease. Curr. Pharm. Des. 22, 6152-6166. doi: 10.2174/1381612822666160907093807

Land, W. G. (2015). The role of damage-associated molecular patterns (DAMPs) in human diseases: part II: DAMPs as diagnostics, prognostics and therapeutics in clinical medicine. Sultan Qaboos Univ. Med. J. 15, e157-e170.

Le, N. T., Narkiewicz, J., Aulić, S., Salzano, G., Tran, H. T., Scaini, D., et al. (2014). Synthetic prions and other human neurodegenerative proteinopathies. Virus Res. 207, 25-37. doi: 10.1016/j.virusres.2014.10.020

Lee, J. W., Lee, Y. K., Yuk, D. Y., Choi, D. Y., Ban, S. B., Oh, K. W., et al. (2008). Neuro-inflammation induced by lipopolysaccharide causes cognitive impairment through enhancement of beta-amyloid generation. J. Neuroinflammation 5:37. doi: 10.1186/1742-2094-5-37

Leshchyn'ka, I., and Sytnyk, V. (2016). Synaptic cell adhesion molecules in Alzheimer's disease. Neural Plast. 2016:6427537. doi: 10.1155/2016/6427537

Li, C. Q., Zheng, Q., Wang, Q., and Zeng, Q. P. (2016). Biotic/abiotic stress-driven Alzheimer's disease. Front. Cell Neurosci. 10:269. doi: 10.3389/fncel.2016.00269

Lim, S. L., Rodriguez-Ortiz, C. J., and Kitazawa, M. (2015). Infection, systemic inflammation, and Alzheimer's disease. Microbes Infect. 17, 549-556. doi: 10.1016/j.micinf.2015.04.004

Lin, C. S., Chang, C. J., Lu, C. C., Martel, J., Ojcius, D. M., Ko, Y. F., et al. (2014). Impact of the gut microbiota, prebiotics, and probiotics on human health and disease. Biomed. J. 37, 259-268. doi: 10.4103/2319-4170. 138314

Lloyd-Price, J., Abu-Ali, G., and Huttenhower, C. (2016). The healthy human microbiome. Genome Med. 8:51. doi: 10.1186/s13073-016-0307-y

Lukiw, W. J. (2012). NF-кB-regulated micro RNAs (miRNAs) in primary human brain cells. Exp. Neurol. 235, 484-490. doi: 10.1016/j.expneurol.2011.11.022

Lukiw, W. J. (2016). Bacteroides fragilis lipopolysaccharide and inflammatory signaling in Alzheimer's disease. Front. Microbiol. 7:1544. doi: 10.3389/fmicb.2016.01544

Lukiw, W. J., and Rogaev, E. I. (2017). Genetics of Aggression in Alzheimer's Disease (AD). Front. Aging Neurosci. 9:87. doi: 10.3389/fnagi.2017.00087

Maldonado, R. F., Sá-Correia, I., and Valvano, M. A. (2016). Lipopolysaccharide modification in Gram-negative bacteria during chronic infection. FEMS Microbiol. Rev. 40, 480-493. doi: 10.1093/femsre/fuw007

Minter, M. R., Taylor, J. M., and Crack, P. J. (2016a). The contribution of neuroinflammation to amyloid toxicity in Alzheimer's disease. J. Neurochem. $136,457-474$.

Minter, M. R., Zhang, C., Leone, V., Ringus, D. L., Zhang, X., OylerCastrillo, P., et al. (2016b). Antibiotic-induced perturbations in gut microbial diversity influences neuro-inflammation and amyloidosis in a murine model of Alzheimer's disease. Sci Rep. 6:30028. doi: 10.1038/srep 30028

Montagne, A., Barnes, S. R., Sweeney, M. D., Halliday, M. R., Sagare, A. P., Zhao, Z., et al. (2015). Blood-brain barrier breakdown in the aging human hippocampus. Neuron 85 296-302. doi: 10.1016/j.neuron.2014. 12.032

Montagne, A., Toga, A. W., and Zlokovic, B. V. (2016). Blood-Brain Barrier Permeability and Gadolinium: Benefits and Potential Pitfalls in Research. JAMA Neurol. 73, 13-14. doi: 10.1001/jamaneurol.2015.2960

Perez, H. J., Menezes, M. E., and d'Acâmpora, A. J. (2014). [Intestinal microbiota]. Acta Gastroenterol. Latinoam. 44, 265-272.

Pinti, M., Appay, V., Campisi, J., Frasca, D., Fülöp, T., Sauce, D., et al. (2016). Aging of the immune system: focus on inflammation and vaccination. Eur. J. Immunol. 46, 2286-2301. doi: 10.1002/eji.201546178

Pistollato, F., Sumalla Cano, S., Elio, I., Masias Vergara, M., Giampieri, F. and Battino, M. (2016). Role of gut microbiota and nutrients in amyloid formation and pathogenesis of Alzheimer disease. Nutr. Rev. 74, 624-634. doi: 10.1093/nutrit/nuw023

Potgieter, M., Bester, J., Kell, D. B., and Pretorius, E. (2015). The dormant blood microbiome in chronic, inflammatory diseases. FEMS Microbiol. Rev. 39, 567-591. doi: 10.1093/femsre/fuv013

Reinus, J. F., and Simon, D. (2014). Gastrointestinal Anatomy and Physiology: The Essentials. New York, NY: Wiley-Blackwell Publishers.

Richards, R. I., Robertson, S. A., O’Keefe, L. V., Fornarino, D., Scott, A., Lardelli, M., et al. (2016). The enemy within: innate surveillance-mediated cell death, the common mechanism of neurodegenerative disease. Front. Neurosci. 10:193. doi: $10.3389 /$ fnins.2016.00193

Rogers, M. A., and Aronoff, D. M. (2016). The influence of non-steroidal anti-inflammatory drugs on the gut microbiome. Clin. Microbiol. Infect. 22, 178.e1-178.e9. doi: 10.1016/j.cmi.2015.10.003

Russo, R., Cristiano, C., Avagliano, C., De Caro, C., La Rana, G., Raso, G. M., et al. (2017). Gut-brain axis: Role of lipids in the regulation of inflammation, pain and CNS diseases. Curr. Med. Chem. doi: 10.2174/0929867324666170216113756. [Epub ahead of print].

Scheperjans, F. (2016). Can microbiota research change our understanding of neurodegenerative diseases? Neurodegener. Dis. Manag. 6, 81-85. doi: 10.2217/nmt-2015-0012

Schwartz, K., Ganesan, M., Payne, D. E., Solomon, M. J., and Boles, B. R. (2016). Extracellular DNA facilitates the formation of functional amyloids in Staphylococcus aureus biofilms. Mol. Microbiol. 99, 123-134. doi: $10.1111 / \mathrm{mmi} .13219$

Sender, R., Fuchs, S., and Milo, R. (2016). Are we really vastly outnumbered? Revisiting the ratio of bacterial to host cells in humans. Cell 164, 337-340. doi: 10.1016/j.cell.2016.01.013

Seong, E., Yuan, L., and Arikkath, J. (2015). Cadherins and catenins in dendrite and synapse morphogenesis. Cell Adh. Migr. 9, 202-213. doi: 10.4161/19336918.2014.994919

Seong, K. J., Lee, H. G., Kook, M. S., Ko, H. M., Jung, J. Y., Kim, W. J. (2016). Epigallocatechin-3-gallate rescues LPS-impaired adult hippocampal neurogenesis through suppressing the TLR4-NF- $\mathrm{kB}$ signaling pathway in mice. Korean J. Physiol. Pharmacol. 20, 41-51. doi: 10.4196/kjpp.2016. 20.1.41

Sharon, G., Sampson, T. R., Geschwind, D. H., and Mazmanian, S. K. (2016) The central nervous system and the gut microbiome. Cell 167, 915-932. doi: 10.1016/j.cell.2016.10.027

Shivaji, S. (2017). We are not alone: a case for the human microbiome in extra intestinal diseases. Gut Pathog. 9, 13. doi: 10.1186/s13099-017-0163-3

Soenen, S., Rayner, C. K., Jones, K. L., and Horowitz, M. (2016). The ageing gastrointestinal tract. Curr. Opin. Clin. Nutr. Metab. Care 19, 12-18. doi: 10.1097/MCO.0000000000000238

Stilling, R. M., Dinan, T. G., and Cryan, J. F. (2014). Microbial genes, brain and behaviour - epigenetic regulation of the gut-brain axis. Genes Brain Behav. 13, 69-86. doi: 10.1111/gbb.12109

Syed, A. K., and Boles, B. R. (2014). Fold modulating function: bacterial toxins to functional amyloids. Front. Microbiol. 5:401. doi: 10.3389/fmicb.2014.00401

Todar, K. (2016). Textbook of Bacteriology. Available online at: http://textbookofbacteriology.net/growth_3.html 
Tremlett, H., Bauer, K. C., Appel-Cresswell, S., Finlay, B. B., and Waubant, E. (2017). The gut microbiome in human neurological disease: a review. Ann. Neurol. 81, 369-382. doi: 10.1002/ana.24901

van de Haar, H. J., Burgmans, S., Jansen, J. F., van Osch, M. J., van Buchem, M. A., Muller, M., et al. (2016). Blood-brain barrier leakage in patients with early Alzheimer disease. Radiology 281, 527-535. doi: 10.1148/radiol.20161 52244

Varatharaj, A., and Galea, I. (2017). The blood-brain barrier in systemic inflammation. Brain Behav. Immun. 60, 1-12. doi: 10.1016/j.bbi.2016.03.010

Zhan, L. S., and Davies, S. S. (2016). Microbial metabolism of dietary components to bioactive metabolites: opportunities for new therapeutic interventions. Genome Med. 8:46. doi: 10.1186/s13073-016-0296-x

Zhan, X., Stamova, B., Jin, L. W., DeCarli, C., Phinney, B., and Sharp, F. R. (2016). Gram-negative bacterial molecules associate with Alzheimer disease pathology. Neurology 87, 2324-2332. doi: 10.1212/WNL.00000000000 03391

Zhao, Y., and Lukiw, W. J. (2015). Microbiome-generated amyloid and potential impact on amyloidogenesis in Alzheimer's disease (AD). J. Nat. Sci. 1:e138.
Zhao, Y., Dua, P., and Lukiw, W. J. (2015). Microbial sources of amyloid and relevance to amyloidogenesis and Alzheimer's disease (AD). J. Alzheimers Dis. Parkinsonism 5:177. doi: 10.4172/2161-0460.1000177

Zhao, Y., Jaber, V., and Lukiw, W. J. (2016). Over-expressed pathogenic miRNAs in Alzheimer's disease (AD) and prion disease $(\operatorname{PrD})$ drive deficits in TREM2-mediated A $\beta 42$ peptide clearance. Front. Aging Neurosci. 8:140. doi: 10.3389/fnagi.2016.00140

Conflict of Interest Statement: The authors declare that the research was conducted in the absence of any commercial or financial relationships that could be construed as a potential conflict of interest.

Copyright (c) 2017 Zhao, Jaber and Lukiw. This is an open-access article distributed under the terms of the Creative Commons Attribution License (CC BY). The use, distribution or reproduction in other forums is permitted, provided the original author(s) or licensor are credited and that the original publication in this journal is cited, in accordance with accepted academic practice. No use, distribution or reproduction is permitted which does not comply with these terms. 